\title{
REAÇÕES ADVERSAS EM USUÁRIAS DE ANTICONCEPCIONAIS ORAIS
}

Adverse reactions in users of oral contraceptives

Efectos adversos en usuarias de anticonceptivos orales

Taciane Christine Siqueira1, Marcelo Del Olmo Sato ${ }^{2}$, Ronise Martins Santiago ${ }^{1 *}$

${ }^{1}$ Centro Universitário Campos de Andrade (UNIANDRADE), Curitiba, Paraná

2 Faculdade Evangélica do Paraná (Fepar), Curitiba, Paraná

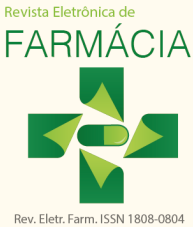

*E-mail: ronise.santiago@gmail.com

\section{RESUMO}

Introdução: Uma das preocupações do uso crônico de anticoncepcionais orais são as reações adversas que podem advir de seu uso, tais como: cefaleia, dores em membros inferiores, sangramento anormal, entre outras. Além disso, as reações adversas são descritas como uma das principais causas de descontinuidade do tratamento.

Objetivo: Dessa forma, o objetivo desta pesquisa, descritiva de corte transversal, foi quantificar as reações adversas relatadas pelas usuárias de anticoncepcionais orais e os motivos para a descontinuidade do tratamento.

Metódos: Para a realização da pesquisa, adotou-se um questionário on-line, destinado a mulheres maiores de 18 anos.

Resultados: Foram analisados 205 questionários respondidos, deste total 86\% de mulheres faziam uso de anticoncepcionais orais e $68,75 \%$ relataram alguma reação adversa durante o uso. Observou-se também que 74,3\% trocaram o anticoncepcional mais de uma vez e $68,7 \%$ descontinuaram o tratamento.

Conclusão: Concluiu-se que os anticoncepcionais orais apresentam reações adversas, como cefaleia, náusea e alterações de humor, capazes de motivar as mulheres a mudarem de anticoncepcional ou descontinuarem o tratamento.

Palavras-chave: Anticoncepcionais orais. Efeitos adversos. Mulheres.

\section{ABSTRACT}

Introduction: One of the concerns of the chronic use of oral contraceptives are the adverse reactions that may result from your use. These can be a headache, pain in the lower limbs, irregular bleeding, among other. In addition to the adverse reactions are described as one of the main cause of discontinuation of treatment.

Objective: Thus, the objective of the study, cross-sectional descriptive, was to quantify adverse reactions reported by users of oral contraceptives and the discontinuation of the treatment.

Methods: Carried out through an online questionnaire, aimed at women older than 18 years.

Results: We analyzed 205 questionnaires answered, this total 86\% made use of oral contraceptives and 68,75\% reported any adverse reaction while using. In addition, $74.3 \%$ changed the pill more than once and $68.7 \%$ discontinued treatment.

Conclusion: It was concluded that the oral contraceptives pose adverse reactions such as headache, nausea and mood swings, which are able to motivate women to change their oral contraceptive or discontinued treatment.

Keywords: Oral contraceptive. Adverse reactions. Women.

\section{RESUMEN}

Introducción: Una de las preocupaciones del uso crónico de los anticonceptivos orales son las reacciones adversas que pueden resultar de su uso. Estas pueden ser una cefalea, dolores en los miembros inferiores, sangrado irregular, entre otras. Además de eso, las reacciones adversas se describen como una de las principales causas de la discontinuidad del tratamiento. 
Objetivo: De esta forma, el objetivo de la investigación, descriptiva de corte transversal, fue cuantificar las reacciones adversas relatadas por las usuarias de anticonceptivos orales y los motivos para la discontinuidad del tratamiento.

Métodos: Para el desarrollo de la investigación, se adoptó un cuestionario en línea, destinado a mujeres mayores de 18 años.

Resultado: Se analizaron 205 cuestionarios respondidos, de ese total 86\% de las mujeres usaban anticonceptivos orales y $68,75 \%$ relataron alguna reacción adversa durante el uso. Además, se observó que el 74,3\% cambió de anticonceptivo más de una vez y el 68,7\% discontinuó el tratamiento.

Conclusion: Se concluyó que los anticonceptivos orales presentan reacciones adversas, como cefalea, náuseas y alteraciones de humor, capaces de inducir a las mujeres a cambiar de anticonceptivo oral o discontinuar el tratamiento.

Palabras clave: Anticonceptivo oral. Efectos adversos. Mujeres.

\section{INTRODUÇÃO}

No Brasil, assim como nos países do terceiro mundo, a divulgação dos métodos contraceptivos, entre eles os anticoncepcionais orais, fazem parte de políticas de saúde voltadas para o controle de natalidade. Enquanto em lugares como a França, onde as pílulas somente foram liberadas para consumo em 1967, no Brasil, tanto elas quanto o dispositivo intrauterino (DIU) são comercializados sem entraves desde o início da década de 1960.(1)

Os anticoncepcionais orais podem ser combinados, contendo em sua formulação dois hormônios (estrógenos e progestógenos) ou podem conter somente o progestógeno, conhecido como minipílulas (2). São o método contraceptivo reversível mais eficiente disponível no mercado e ao mesmo tempo o mais utilizado no mundo ${ }^{(3)}$, representando a melhor opção em termos de êxito contraceptivo, quando não há falha na ingestão das pílulas. Seu mecanismo de ação reside na inibição da ovulação pelo bloqueio do pico do hormônio luteinizante (LH); por modificações do endométrio, dificultando a nidação; por alterações no muco cervical, tornando-o hostil à ascensão dos espermatozóides; por modificações no peristaltismo tubário, interferindo no transporte ovular e causando alterações na resposta ovariana às gonadotropinas hipofisárias ${ }^{(4)}$.

Por outro lado, as reações adversas ao medicamento, definidas pela Organização Mundial da Saúde como "qualquer resposta prejudicial ou indesejável e não intencional que ocorre com medicamentos em doses normalmente utilizadas no homem para profilaxia, diagnóstico, tratamento de doença ou para modificação de funções fisiológicas" (5), podem ser observadas entre usuárias desse método, sendo muitas vezes motivo para o abandono do tratamento contraceptivo. ${ }^{(6)}$ Essa descontinuação do anticoncepcional oral pode ocasionar principalmente a gravidez não planejada ou não desejada. ${ }^{(3)}$

Além disso, a elevação na quantidade de gestações não planejadas traz como consequência o aumento dos índices de aborto induzido. Aproximadamente $50 \%$ das mulheres que residem nas regiões Sul e Sudeste, e que praticaram o aborto, declararam utilizar métodos contraceptivos. ${ }^{(7)}$

Dessa forma, o presente estudo teve como objetivo quantificar as principais reações adversas observadas pelas usuárias e a influência na descontinuidade do tratamento.

\section{MATERIAIS E MÉTODOS}

A pesquisa descritiva de corte transversal foi realizada por meio de questionário on-line, elaborado no Google Drive, no período entre 25 de agosto a 25 de setembro de 2016. O trabalho foi submetido ao Comitê de ética em Pesquisa da Uniandrade e aprovado de acordo com o parecer número 1.710.734. 
A participante, voluntária, para iniciar o questionário tinha que obrigatoriamente aceitar o termo de conhecimento livre e esclarecido (TCLE). Somente após o aceite, o acesso ao questionário, semiestruturado e autoaplicável, era permitido. Com ele, analisou-se o perfil farmacoepidemiológico das usuárias, as reações adversas causadas pelo anticoncepcional oral e os motivos para a descontinuidade do tratamento. O questionário foi composto de perguntas objetivas ou discursivas, dividido em duas partes. Na primeira parte havia perguntas para caracterizar a amostra, como: idade, tempo de uso, métodos contraceptivos, uso de contraceptivo oral e o nome do contraceptivo que utilizava. A segunda parte do questionário (respondido somente por mulheres que utilizavam o remédio) eram perguntas para avaliar as reações adversas e causa de descontinuidade. As indagações foram: qual pílula utilizava na época da reação adversa, se já trocou o fármaco ou mesmo parou de usar e por quê; se possui alguma doença considerada contraindicada para o uso do medicamento, se era fumante ou não. O questionário foi escrito em linguagem simples (sem uso de termos técnicos) para facilitar o entendimento de todas as participantes.

A estratégia de divulgação, por meio de redes sociais, permitiu a inclusão de mulheres de diferentes idades e estratos socioeconômicos. Foram excluídas da pesquisa mulheres com idade inferior a 18 anos e também aquelas que se recusaram a aceitar o TCLE ou a responder ao questionário. Os dados foram analisados e expressos em porcentagem ou frequência absoluta.

\section{RESULTADOS}

A amostra do trabalho foi composta por 205 questionários respondidos. Entre as participantes, a idade mínima foi de 18 e a máxima de 51 anos, sendo a faixa etária entre 23 e 25 anos a mais predominante. Do total, 152 entrevistadas utilizavam pílula anticoncepcional.

Duzentas e cinco mulheres foram entrevistadas, $85,9 \%$ delas faziam uso de algum tipo de contraceptivo e apenas $14,1 \%$ não utilizavam nenhum tipo. Quanto ao método utilizado, o anticoncepcional oral foi o mais utilizado, representando $86,4 \%$ da amostra, seguido do anticoncepcional injetável com 5,7\% e do DIU com 4,0\%. A camisinha representou 2,8\% das entrevistadas e outros 1,1\% de mulheres que utilizam tabelinha e anel vaginal (Gráfico 1).

Figura 1. Porcentagem dos métodos contraceptivos utilizados pelas mulheres entrevistada

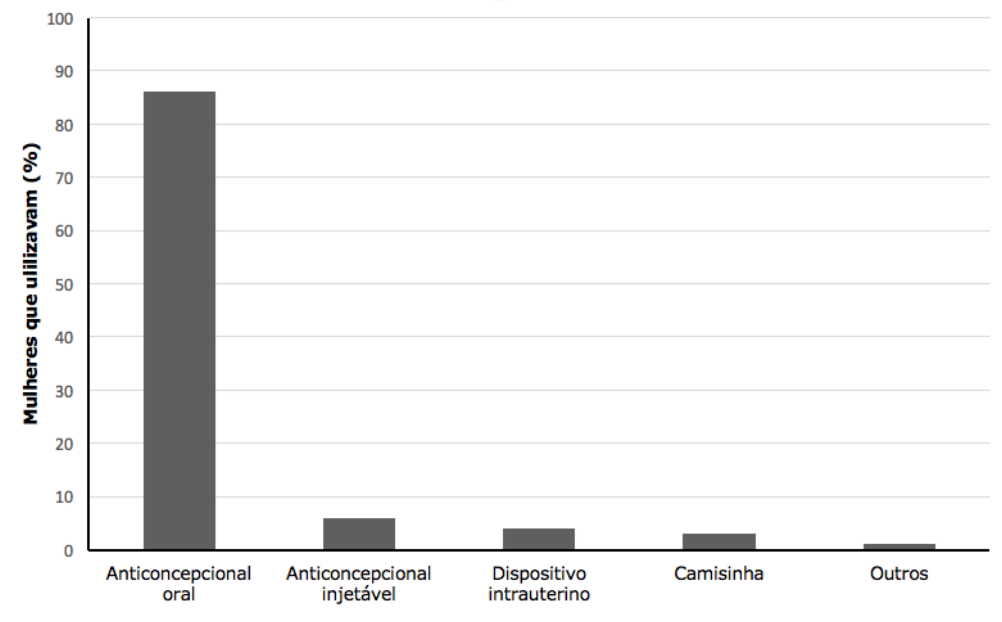


Em relação ao tempo de uso dos anticoncepcionais orais, demonstrado na Gráfico 2, constatamos que 70,4\% das entrevistadas os utilizam há mais de quatro anos, 12,5\% há três anos, 6,3\% há dois anos 7,4\% há um ano e 3,4\% há menos de um ano.

Gráfico 2. Tempo de uso, em anos, do anticoncepcional oral

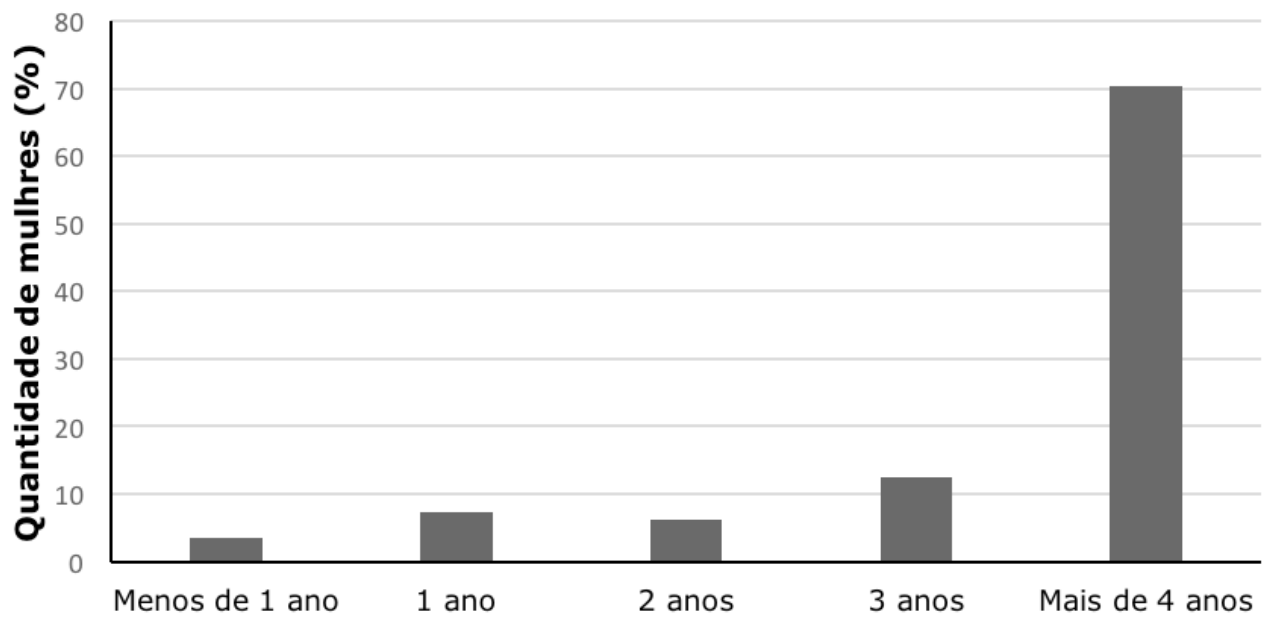

Entre os medicamentos mais utilizados destacaram-se o Diane 35 (composto pelos princípios ativos acetato de ciproterona $2 \mathrm{mg}$ + etinilestradiol 0,035 mg), o Elani Ciclo, o Yasmim que contêm os mesmos hormônios (3 mg de drospirenona e 0,03 mg de etinilestradiol) e o Yaz (3 mg de drospirenona e 0,02 mg de etinilestradiol). No total foram citados 17 compostos diferentes (ou principio ativo das pílulas), entretanto ao considerar a marca comercial o resultado foi de 40 remédios diferentes (Tabela 1).

Tabela 1. Anticoncepcionais orais utilizados pelas entrevistadas

\begin{tabular}{l|c|l}
\hline Composição do Anticoncepcional oral & $\begin{array}{c}\text { Número } \\
\text { de } \\
\text { usuárias }\end{array}$ & Nome comercial do Anticoncepcional oral \\
\hline $2 \mathrm{mg}$ de acetato de ciproterona $+0,035 \mathrm{mg}$ de etinilestradiol & 21 & Diane 35; Selene \\
\hline $3 \mathrm{mg}$ de drospirenona $+0,03 \mathrm{mg}$ de etinilestradiol & 20 & Yasmin; Elani 28; Moliere \\
\hline $3 \mathrm{mg}$ de drospirenona $+0,02 \mathrm{mg}$ de etinilestradiol & 17 & Yaz; Lumi \\
\hline $0,15 \mathrm{mg}$ de levonorgestrel $+0,03 \mathrm{mg}$ de etinilestradiol & 16 & Ciclo 21; Microvilar; Nociclin \\
\hline $3 \mathrm{mg}$ de drospirenona $+0,03 \mathrm{mg}$ de etinilestradiol & 14 & Elani Ciclo \\
\hline $0,075 \mathrm{mg}$ de gestodeno $+0,02 \mathrm{mg}$ de etinilestradiol & 14 & $\begin{array}{l}\text { Alestra 20; Diclin; Diminut; Ginese; } \\
\text { Micropil 20; Harmonete; Previane }\end{array}$ \\
\hline $0,075 \mathrm{mg}$ de desogestrel & 11 & Cerazette; Desogestrel \\
\hline
\end{tabular}




\begin{tabular}{|c|c|c|}
\hline $0,060 \mathrm{mg}$ de gestodeno $+0,015 \mathrm{mg}$ de etinilestradiol & 10 & Tâmisa 20; Siblima; Tantin; Minesse; Mínima \\
\hline $0,15 \mathrm{mg}$ de desogestrel $+0,020 \mathrm{mg}$ de etinilestradiol & 9 & Femina; Primera; Mercilon Conti \\
\hline $\begin{array}{l}3 \mathrm{mg} \text { de estradiol e } 2 \mathrm{mg} \text { de estradiol }+2 \mathrm{mg} \text { dienogeste e } \\
2 \mathrm{mg} \text { de estradiol }+3 \mathrm{mg} \text { dienogeste e } 1 \mathrm{mg} \text { de estradiol e } \\
\text { pílulas inativas sem hormônio }\end{array}$ & 8 & Qlaira \\
\hline $0,075 \mathrm{mg}$ de gestodeno $+0,03 \mathrm{mg}$ de etinilestradiol & 8 & Gestinol 28; Tamisa 30; Allestra 30; Minulet \\
\hline $2 \mathrm{mg}$ de acetato de clormadinona $+0,03 \mathrm{mg}$ de etinilestradiol & 4 & Belara \\
\hline $\begin{array}{l}0,025 \mathrm{mg} \text { de I-desogestrele }+0,04 \mathrm{mg} \text { de etinilestradiol e } \\
0,125 \mathrm{mg} \text { de desogestrel }+0,03 \mathrm{mg} \text { etinilestradiol }\end{array}$ & 3 & Gracial \\
\hline $0,10 \mathrm{mg}$ de levonorgestrel $+0,02 \mathrm{mg}$ etinilestradiol & 2 & Level \\
\hline $0,15 \mathrm{mg}$ de desogestrel $+0,03 \mathrm{mg}$ de etinilestradiol & 2 & Microdiol \\
\hline $2,5 \mathrm{mg}$ de acetato de nomegestrol $+1,5 \mathrm{mg}$ de estradiol & 2 & Stezza \\
\hline $0,15 \mathrm{mg}$ de levonorgestrel $+0,03 \mathrm{mg}$ de estradiol & 1 & Nordete \\
\hline $\begin{array}{l}0,05 \mathrm{mg} \text { de levonorgestrel }+0,03 \mathrm{mg} \text { de etinilestradiol e } \\
0,075 \mathrm{mg} \text { de levonorgestrel }+0,04 \mathrm{mg} \text { de etinilestradiol e } \\
0,125 \mathrm{mg} \text { de levonorgestrel }+0,03 \mathrm{mg} \text { de etinilestradiol }\end{array}$ & 1 & Triquilar \\
\hline
\end{tabular}

Sobre as principais reações adversas, $68,7 \%$ das mulheres relataram apresentar ou já terem apresentado alguma reação adversa com o uso de anticoncepcionais orais. Em número de entrevistadas, as reações mais comuns foram: cefaleia $(n=57)$, náusea ( $n=38)$, alteração de humor $(n=37)$, ganho de peso $(n=34)$, sangramento anormal $(n=27)$, acne $(n=18)$, tontura $(n=16)$, tromboembolismo pulmonar (TEP; $n=2$ ) e outros como, por exemplo, inchaço nas pernas ( $n=2)$ (Tabela 2), todas faziam uso de pílula combinada. Entre as duas mulheres que marcaram a opção TEP, uma relatou que estava em tratamento, entretanto não descreveu a conduta adotada pelo médico ou o medicamento utilizado para o tratamento, por outro lado o anticoncepcional utilizado por elas era o Yasmim. Em relação ao restante das mulheres que relataram reação adversa, os dados demonstraram que o Diane 35 e o Yasmim eram os mais utilizados.

Tabela 2. Reações Adversas relatadas pelas mulheres que utilizam anticoncepcional oral

\begin{tabular}{c|c}
\hline Reação adversa & Número de mulheres \\
\hline Dor de cabeça & 57 \\
\hline Enjoo & 38 \\
\hline Alteração do humor & 37 \\
\hline Ganho de peso & 34 \\
\hline Sangramento inesperado & 27 \\
\hline Acne & 18 \\
\hline Tontura & 16 \\
\hline Tromboembolismo pulmonar (TEP) & 2 \\
\hline Outros & 2 \\
\hline
\end{tabular}


Ao serem questionadas sobre quantas vezes já trocaram de medicamento, constatou-se que $74,3 \%$ das mulheres que utilizavam anticoncepcional oral mudaram de fármaco. O resultado foi dividido da seguinte forma: $42,8 \%$ mudaram de pílula pelo menos uma vez, 23,7\% de duas a três vezes e 7,9\% quatro ou mais vezes, como demonstrado no Gráfico 3 .

Gráfico 3. Porcentagem de mulheres que já trocaram de anticoncepcional oral de acordo com a quantidade de substituição

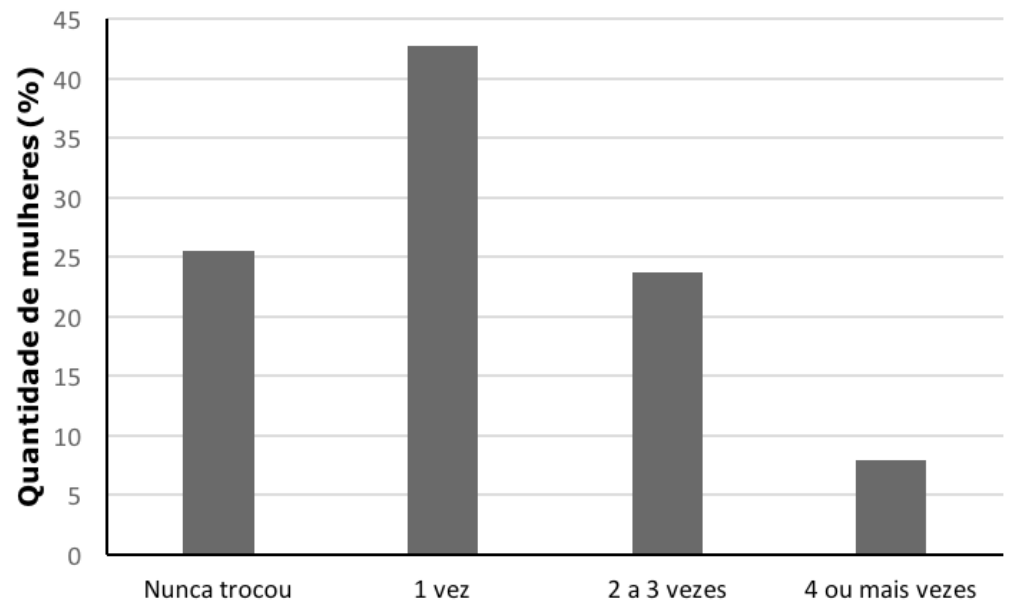

A respeito do motivo da substituição, 26,3\% mulheres fizeram por indicação de amigo, 25,0\% por reação adversa, 7,9\% por não obterem o resultado esperado, 4,6\% devido ao preço e outros fatores como amamentação. Retenção de líquido e risco à saúde também foram citados por $4,6 \%$ das mulheres (Gráfico 4).

Gráfico 4. Motivo da mudança de pílula relatado pelas entrevistadas

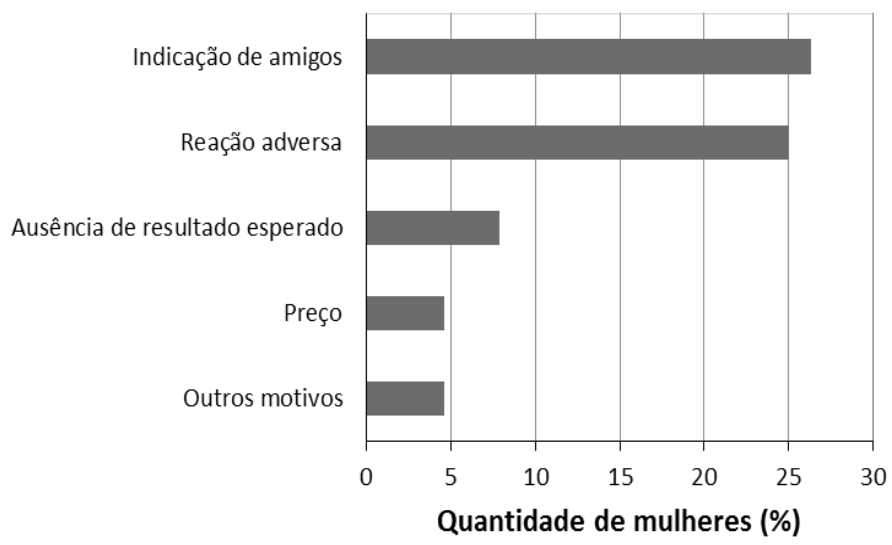


O Gráfico 5 demonstra por quanto tempo as mulheres interromperam o uso do medicamento. De acordo com os dados, $72,4 \%$ delas descontinuaram o tratamento por algum tempo. Entre as causas para interromper a utilização do fármaco, constatamos que a vontade de engravidar $(24,5 \%)$ foi a mais citada pelas entrevistadas, seguida por reação adversa $(23,6 \%)$, esquecimento $(20,0 \%)$ e outros motivos como cirurgia $(20 \%)$, gravidez inesperada $(11,8 \%)$ e preço $(7,2 \%)$.

Figura 5. Motivo da descontinuidade do tratamento com o anticoncepcional oral

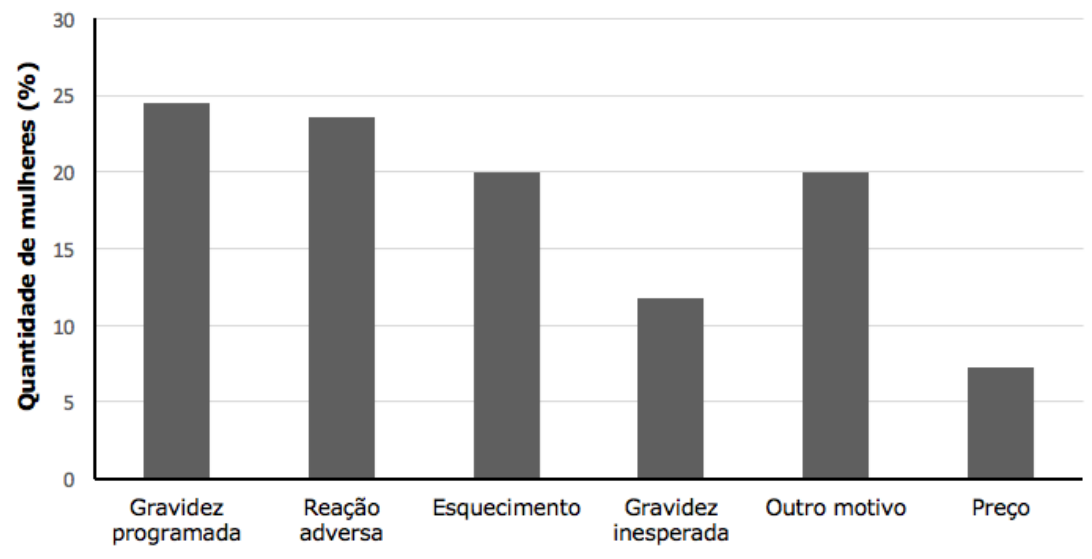

Por fim, em relação ao fato das entrevistadas possuírem doenças que contraindicam o uso de anticoncepcional oral, 25 mulheres responderam que possuem ou apresentaram varizes, 22 sangramentos anormais, 26 com colesterol alto, 4 com problemas cardíacos; outras doenças como ovário policístico e hipotireoidismo foram relatadas por 6 entrevistadas (Gráfico 6). Em relação ao fato das entrevistadas serem fumantes ou não, apenas 2,8\% marcaram a opção sim.

Figura 6. Patologias apresentadas pelas mulheres que utilizam os anticoncepcionais orais

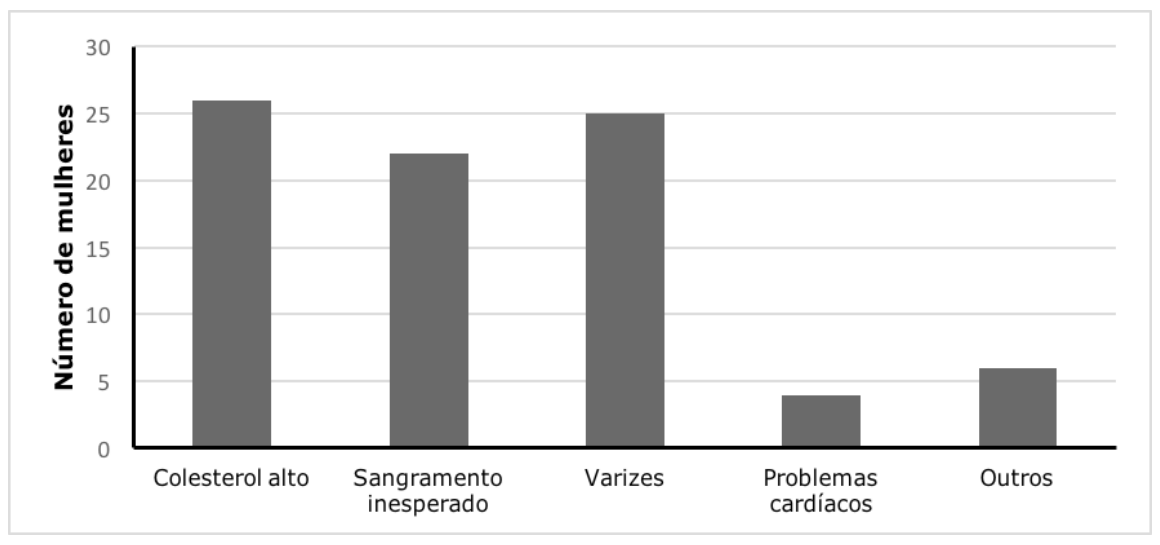




\section{DISCUSSÃO}

Este estudo demonstrou que 85,9\% das participantes, com idade predominante entre 23 a 25 anos, utilizavam algum tipo de método contraceptivo e $86,36 \%$ delas usavam anticoncepcionais orais. Além disso, a maioria das mulheres (70,45\%) utiliza o método há mais de 4 anos. Entre os remédios mais empregados, destacamos: o Diane 35, composto por acetato de ciproterona $2 \mathrm{mg}$ + etinilestradiol 0,035mg e o Elani Ciclo com 3mg de drospirenona e 0,03mg de etinilestradiol. Dentre as reações adversas, as mais citadas foram: alteração de humor, dor de cabeça e náusea. Em relação a 152 mulheres que usam a pílula, 74,3\% trocaram de medicamento mais de uma vez e 68,7\% descontinuaram o tratamento. Assim, devido às reações adversas, $25 \%$ delas substituíram o fármaco e $23,6 \%$ interromperam o uso.

O uso de anticoncepcionais orais é contraindicado em algumas situações, pelo fato de aumentarem a probabilidade de AVC, TEP e problemas cardíacos: 25 mulheres relataram apresentar varizes e 26 tiveram colesterol alto. Já as fumantes representaram apenas $2,8 \%$ da amostra.

Os compostos estrogênicos são os principais responsáveis pelas reações adversas dos fármacos, tais como o tromboembolismo e a piora da migrânea, sendo que, quanto maior a dose dos estrógenos, maiores são esses efeitos. ${ }^{(8)}$ Neste estudo, a maioria das entrevistadas usava anticoncepcionais orais combinados (92,8\% da amostra).

As reações adversas têm sido apontadas como a principal causa de descontinuação de uso de pílula anticoncepcional. Neste trabalho, as reações adversas mais citadas foram: a alteração de humor, cefaleia, náusea, ganho de peso e sangramento anormal. Além disso, 23,6\% das mulheres relataram que descontinuaram o anticoncepcional e $25 \%$ trocaram o medicamento devido a reações adversas. Entretanto, não correlacionamos a taxa de descontinuação com o tipo de remédio utilizado pela entrevistada.

O risco da mulher que apresenta náuseas, sangramento inesperado ou mastalgia de interromper o tratamento é duas vezes maior do que aquela sem sintomas ${ }^{(9)}$. Nossos dados são ainda corroborados por outros estudos, de acordo com Rosenberg e colaboradores ${ }^{(10)}$, nos Estados Unidos, 37\% das usuárias já interromperam o uso de anticoncepcional oral por apresentarem reação adversa. Em um estudo de corte transversal realizado em diversos países, incluindo o Brasil, a taxa de descontinuação ou troca por outro método devido a reações adversas foi de $57 \%{ }^{(11)}$. Uma investigação realizada com 1.427 mulheres, demonstrou que $40 \%$ das descontinuações aconteceram por cefaléia, aumento de peso e sangramento uterino irregular ${ }^{(3)}$. Os dados demonstraram também que no Brasil $45 \%$ das usuárias de pílula descontinuam o uso no primeiro ano, sendo $12 \%$ por reações $\operatorname{adversas}^{(12)}$.

Segundo Correia e colaboradores ${ }^{(13)}$, em média 20,0\% das mulheres brasileiras apresentaram alguma condição que contraindica o uso de anticoncepcional. Em seu estudo, com 20.454 mulheres, 11,7\% das usuárias de pílula não deveriam utilizá-lo. A principal circunstância para a contraindicação é a Hipertensão Arterial Sistêmica (HAS), seguida da idade maior que 35 anos, concomitante ao tabagismo.

Os acidentes vasculares celebrais estão diretamente relacionados à HAS e com maior chance de ocorrência ao fazer uso de fármaco à base de estrogênios ${ }^{(14)}$. O uso de cigarro também não é recomendado, pois pode levar a problemas cardíacos graves, como o infarto agudo do miocárdio, quando associados ao uso do medicamento ${ }^{(8,15)}$. Desse modo, as pílulas são contraindicadas para mulheres que tenham ou já tiveram qualquer distúrbio tromboembólico, doença vascular cerebral ou arterial, problemas cardíacos, câncer de mama (mesmo que seja apenas uma suspeita), tumor hepático benigno ou maligno ou outra disfunção hepática, hiperlipidemia congênita e sangramento vaginal anormal não diagnosticado, idade acima dos 35 anos e fumantes(16). Mulheres hipertensas, fumantes ou com idade superior a 35 anos estão mais propensas a ter um acidente vascular encefálico (AVE) isquêmico e hemorrágico. ${ }^{(17)}$

O risco de eventos tromboembólicos relacionado ao tipo de anticoncepcionais orais é de 5,6 vezes mais para aqueles que contêm gestodeno; 7,3 vezes para desogestrel; 6,8 vezes para acetato de ciproterona e 6,3 vezes para drospirenona em 
comparação a mulheres que não utilizam o remédio. O mesmo estudo indica que os anticoncepcionais com ação antiandrogênica (como, por exemplo, o Diane, Yasmin e Yaz) apresentam um risco quatro vezes maior para tromboembolismo venoso em comparação com outras pílulas (18). Ainda de acordo com a ANVISA, os que precisam de maior atenção, pelo risco de ocasionar eventos trombóticos, são o Diane 35 e o Yasmin ${ }^{(19)}$. Neste trabalho, as duas entrevistadas que relataram ter tido TEP estavam utilizando o anticoncepcional oral com os princípios ativos drospirenona e etinilestradiol.

O trabalho apresenta algumas limitações, uma delas é o fato de não termos utilizado algoritmos de casualidade. Outro fator é número de participantes: como o questionário foi disponibilizado por meio de redes sociais e aplicativos de mensagem, a quantidade de entrevistadas foi dependente do número de mulheres que o receberam e optaram por respondê-lo.

\section{CONCLUSÃO}

Conclui-se que a presença de reações adversas como cefaleia, náusea e alteração de humor devido ao uso de anticoncepcionais orais, pode induzir as mulheres a trocarem ou descontinuarem seu tratamento contraceptivo.

\section{REFERÊNCIAS}

1. Pedro JM. A experiência com contraceptivos no Brasil: uma questão de geração; Revista Brasileira de História. 2003; 23(45): 239-260.

2. Wannmacher L. Anticoncepcionais orais: $\mathrm{O}$ que há de novo. Uso racional de medicamentos: temas selecionados. Brasília. 2003; 1: 4-5.

3. Bahamondes L, Pinho F, Melo NR, Oliveira E, Bahamondes MV. Fatores associados à descontinuação do uso de anticoncepcionais orais combinados. Revista Brasileira de Ginecologia e Obstetrícia. 2011; 33: 303-309.

4. Pereira SM, Taquette SR. Desvendando mitos sobre anticoncepção hormonal oral na adolescência. Adolescência e Saúde. 2008; 5: 45-49.

5. WHO. World Health Organization. The Uppsala Monitoring Centre. The Importance of Pharmacovigilance. Safety Monitoring of medicinal products. 2002.

6. Pereira SM, Taquette SR. Desvendando mitos sobre anticoncepção hormonal oral na adolescência. Adolescência e Saúde. 2008; 5: 45-49.

7. Brasil. Ministério da Saúde. Aborto e saúde pública no Brasil: 20 anos. Brasília: Ministério da Saúde, 2009.

8. Giglio MRP, Andrade LC, Daher GM, Ribeiro MO, Albernaz MA. Contracepção Hormonal segundo a Ótica do Estudante de Medicina: Mais um Desafio para o Ensino Médico Brasileiro? Rev. bras. educ. Med. 2015; 39(4): 502-506.

9. Rosenberg MJ, Waugh MS, Meehan TE. Use and misuse of oral contraceptives: risk indicators for poor pill taking and discontinuation. Contraception 1995; 51(5):283-8.

10. Rosenberg MJ, Waugh MS. Oral contraceptive discontinuation: a prospective evaluation of frequency and reasons. Am. J. Obstet. Gynecol. 1998; 179:577-82.

11. Hooper DJ. Attitudes, awareness, compliance and preferences among hormonal contraception users: a global, crosssectional, self-administered, online survey. Clin. Drug. Investig. 2010; 30(11): 749-63. 
12. Leite IC. Descontinuação de métodos anticoncepcionais no Nordeste do Brasil, 1986-1991. Caderno de Saúde Pública. Rio de Janeiro. 2003; 19(4): 1005-1016.

13. Corrêa DAS, Felisbino-Mendes MS, Mendes MS, Malta DC, Velasquez-Melendez G. Fatores associados ao uso contraindicado de contraceptivos orais no Brasil. Rev. Saúde Pública. 2017; 51:1.

14. Rosa CM, Coutinho KS, Domingues MF, Moura DS. A prevalência de acidente vascular cerebral (AVC) no pós-operatório de revascularização do miocárdio. Revista Brasileira em Promoção da Saúde. 2006; 19(1): 25-34.

15. Lemos ERL, Moura ERF, Guedes TG, Nogueira PSF, Souza CBJ. Uso de métodos anticoncepcionais por mulheres assistidas em área distrital e fatores determinantes. Revista de APS. 2001; 14(4): 417-423.

16. Lupidão AC, Okazaki ELFJ. Métodos anticoncepcionais: revisão. Rev. Enferm. UNISA. 2011; 12(2): $136-141$.

17. Almeida APF, Assis MM. Efeitos colaterais e alterações fisiológicas relacionadas ao uso contínuo de anticoncepcionais hormonais orais. Rev. Eletrôn. Atualiza Saúde 2017; 5(5): 85-93.

18. Vlieg AH, Helmerhorst FM, Vandenbroucke JP, Doggen CJM, Rosendaal FR. The venous thrombotic risk of oral contraceptives, effects of oestrogen dose and progestogen type: results of the MEGA-case control study. BMJ. 2009; 339; 2921.

19. Brasil - Agência Nacional de Vigilância Sanitária. Informe SNVS/Anvisa/Nuvig/GFARM nº1. Brasília, 2013. Disponível em: <http://ansm.sante.fr/S-informer/Actualite/Diane-R-35-et-ses-generiques-Communique>. Acessado em 11/06/2017.

Submetido em: 18/02/2017 